\title{
The incidence rate of pituitary adenomas in western Sweden for the period 2001-2011
}

\author{
Axel Tjörnstrand ${ }^{1,{ }^{*},+}$, Kerstin Gunnarsson ${ }^{1, *}$, Max Evert ${ }^{1,{ }^{*}}$, Erik Holmberg ${ }^{2}$, \\ Oskar Ragnarsson ${ }^{1,3}$, Thord Rosén ${ }^{1,3}$ and Helena Filipsson Nyström ${ }^{1,3}$ \\ ${ }^{1}$ Sahlgrenska Academy and ${ }^{2}$ Department of Oncology, Institute of Clinical Sciences, Sahlgrenska Academy, \\ University of Gothenburg, Gothenburg, Sweden and ${ }^{3}$ Department of Endocrinology, Sahlgrenska University \\ Hospital, Gothenburg, Sweden \\ *(A Tjörnstrand, K Gunnarsson and M Evert contributed equally to this work) \\ ${ }^{\dagger} A$ Tjörnstrand is now at Department of Endocrinology, Sahlgrenska University Hospital, Blå Stråket 5, \\ SE-413 45 Göteborg, Sweden
}

Correspondence
should be addressed
to A Tjörnstrand
Email
axel.tjornstrand@vgregion.se

\begin{abstract}
Objective: The number of studies on the incidence of pituitary adenomas (PAs) is limited. The aim of this study was to evaluate the standardised incidence rate (SIR) of PAs in western Sweden.

Design, subjects and methods: Data from adult patients diagnosed with PAs in 2001-2011, living in the Västra Götaland County, were collected from the Swedish Pituitary Registry (SPR). In addition, medical records on all patients diagnosed with PAs at the six hospitals in the region were reviewed. In total, 592 patients were included in the study.

Age-SIR, given as rate/100 000 inhabitants $(95 \% \mathrm{CI})$, was calculated using the WHO 2000 standard population as a reference. Results: The total SIR for PAs was 3.9/100 000 (3.6-4.3); 3.3/100 000 (2.9-3.7) for men and 4.7/100 000 (4.1-5.3) for women. In men, SIR increased with age, while in women SIR peaked at 25-34 years, mainly due to prolactinomas. Non-functioning PA (NFPA) was the most common PA (54\%, 1.8/100 000 (1.6-2.0)) followed by prolactinomas (32\%, 1.6/100 000 (1.3-1.9)), acromegaly (9\%, $0.35 / 100000$ (0.25-0.45)), Cushing's disease (4\%, 0.18/100 000 (0.11-0.25)) and TSH-producing PA (0.7\%, 0.03/100 000 (0.00-0.05)). The proportion of macroadenomas for NFPA was $82 \%$, prolactinomas $37 \%$, GH-producing PA $77 \%$, ACTH-producing PA $28 \%$ and TSH-producing PA $100 \%$. The lifetime risk for PAs was $0.27 \%(0.24-0.31)$ in men and $0.29 \%(0.26-0.33)$ in women.

Conclusion: This study provides a reliable estimate on the overall incidence of PAs and confirms an increased incidence of PAs compared with studies conducted in the pre-magnetic resonance imaging era. The lower proportion of prolactinomas compared with previous studies is probably explained by the different criteria used.
\end{abstract}

\section{Introduction}

The number of studies on the incidence of pituitary adenomas (PAs) is limited. In 1958, the incidence of PAs was reported to be 0.6 cases/100 000 per year and, in 1991, 1.1 cases/100 000 per year (1). Not all cases of PAs become clinically evident. In post-mortem and imaging studies, incidentally discovered PAs are detected in $10-11 \%$ of cases $(2,3,4,5,6)$. In a meta-analysis published in 2004, the prevalence of PAs was $14.4 \%$ in post-mortem studies and $22.5 \%$ in radiology studies (7).

Therefore, it seems reasonable to assume that the detection of PAs has increased with the introduction of magnetic resonance imaging (MRI). This has in fact been observed in a recent incidence study from northern Finland. In 1992-2007, all PAs collected from a welldefined cohort of 730000 inhabitants in northern Finland had a standardised incidence rate (SIR) of four cases/100 000 per year (8), which was higher than that reported previously (1).

The performance of incidence and prevalence studies in Sweden is facilitated by the organisation of the Swedish healthcare system, which is government funded and managed at national, regional and local levels.
(ㄷ) 2014 European Society of Endocrinology Printed in Great Britain
Published by Bioscientifica Ltd. 


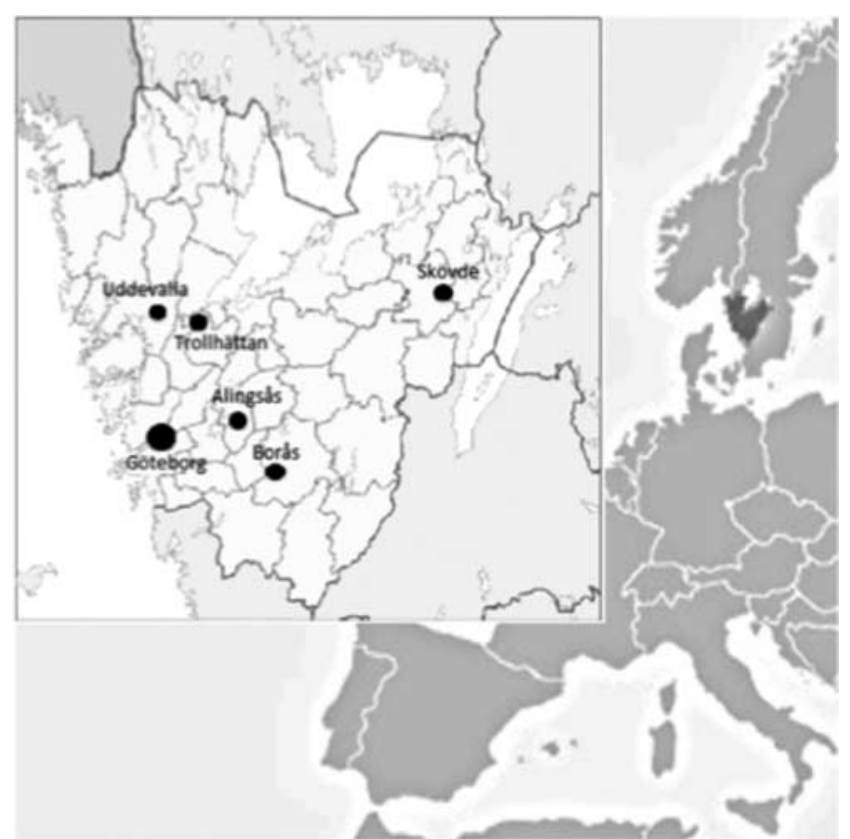

\section{Figure 1}

Map over the Västra Götaland County in Sweden and its hospitals.

There are six healthcare regions, each with at least one university hospital with specialist care in pituitary diseases and neurosurgery. A unique personal number identifies every patient and all visits are coded in a diagnosis-related group (DRG) registry according to the International Statistical Classification of Diseases and Related Health Problems 10th revision (ICD-10). This organisation offers an ideal opportunity for epidemiological research.

The Västra Götaland (VG) County is located in the southwest of Sweden (Fig. 1) and has a well-defined population of 1.6 million inhabitants (Statistics, Sweden) and one university hospital and five local hospitals with endocrine departments. The majority of patients with large tumours and hormone-producing tumours are referred to the university hospital, whereas patients with smaller PAs and prolactinomas are often treated at the local hospital or gynaecologist.

The primary aim of this study was to evaluate the standard incidence rate (SIR) of PAs between 2001 and 2011 in the VG County during a period with a frequent use of MRI. Secondary aims were to estimate SIR of the different sub-types of PAs, to analyse time trends and to calculate the lifetime risk.

\section{Patients and methods}

\section{Study design}

This study was based on data from the Swedish Pituitary Registry (SPR; http://www.svenskahypofysgruppen.se/ hypofysregistret.html). SPR is a national quality registry, established in 1991, where information on basic demography, clinical findings, biochemical analyses, radiology, histopathology, treatment and outcome in patients with PAs is collected. Data are registered at diagnosis, in connection with primary treatment (surgery and/or radiotherapy), 1 year after treatment, and then every fifth year after treatment. This registration is usually performed by an endocrinologist operating in the public healthcare. During the study period, no private endocrinologist was practising in the VG region. The SPR does not have a 100\% cover rate. In order to better evaluate the true incidence of PAs, the authors went through the medical records of every patient diagnosed with a PA between 2001 and 2011 after searching the DRG registry from this period. Patients already registered in the SPR were re-evaluated and, in the case of missing or incomplete data, the additional information was added. Moreover, patients who were not identified in the SPR were added to the register if PA was confirmed. Thereby, a uniform evaluation for all patients is provided in the registry. However, the SPR does not collect information on whether the tumours are incidentally discovered or not. Patients with PAs fulfilling the following predefined criteria were eligible for the study. Non-functioning PA (NFPA) was defined as an adenoma, $\geq 3 \mathrm{~mm}$ on MRI/CT, without clinical or laboratory evidence of excessive hormone production. Therefore, adenomas containing gonadotrophic or null-null cells on histological examination were classified as NFPA. Adenomas that were adrenocorticotrophic hormone (ACTH), growth hormone (GH) and/or gonadotroph positive immunohistochemically, but without evident hormonal secretion, were classified as NFPA. The diagnosis of prolactinomas was based on clinical findings, radiological identification of PAs and biochemical analysis of prolactin in serum. The initial serum prolactin level was missing in some patients, where treatment with dopamine agonists was initiated by private gynaecologists. These patients were considered to have prolactinomas if a subsequent MRI confirmed demonstrated PAs. Patients with hyperprolactinaemia but no visible adenoma were included if prolactin levels exceeded three times the upper limit of the normal reference range, and no other cause for the hyperprolactinaemia could be identified. There were 24 reported prolactinomas that were 
undetectable on MRI or smaller than $3 \mathrm{~mm}$. Information on MRI findings in ten patients with prolactinomas was unavailable. The diagnoses of ACTH-, GH- and thyroidstimulating hormone (TSH)-producing PAs were based on clinical, biochemical and radiological data. In addition, in patients with TSHomas, the diagnosis was based on the response of TSH and $\alpha$-subunit to thyrotrophin-releasing hormone and/or the triiodothyronine suppression test. Histopathological diagnosis is not a prerequisite for inclusion in the SPR, instead individuals were included based on the clinical presentation of PAs.

Adenomas secreting more than one hormone were classified as the subgroup with the most rare hormonal production, in accordance with the Finnish study (8), i.e. $\mathrm{TSH}>\mathrm{ACTH}>\mathrm{GH}>\mathrm{PRL}$. Macroadenomas were defined as tumours $\geq 1 \mathrm{~cm}$ in diameter and microadenomas were defined as tumours $<1 \mathrm{~cm}$. Cases with craniopharyngeomas and secondary pituitary tumours, i.e. metastases, were not included in the study.

\section{Patient population}

Patients diagnosed with PAs between 2001 and 2011 in VG County were included in this study. The population register for VG County was used to confirm that the patients were resident in the county. The population in VG County during this study period was 1.6 million (1 590640 at the end of the study period).

In addition to the data from the SPR, a search in the DRG registries at the Departments of Medicine in Alingsås, Borås, Skövde, Uddevalla, Trollhättan and Göteborg (Fig. 1) was conducted with the ICD-10 codes for benign neoplasm of the pituitary gland (D35.2), acromegaly and pituitary gigantism (E22.0), hyperprolactinaemia (E22.1), Cushing's disease (E24.0) and TSHoma (E05.8). If a PA was confirmed, patients who were not previously included in the SPR were also registered. After completed registration, the information on patients with PAs fulfilling the inclusion criteria was collected from the SPR.

Besides inclusion in the SPR, patients were eligible for inclusion in the study if the diagnosis was established after the age of 18 years, the patients were living in VG County at the time of diagnosis, and the diagnosis was established between 2001 and 2011.

\section{Ethics}

The SPR was approved by the Ethics Committee (675/03 and 2012/915-32) in Stockholm, Sweden. This study was performed according to the Declaration of Helsinki.

\section{Statistical analysis}

The analyses of the PA incidence rate were based on the assumption of a Poisson distribution. The incidence rate was age standardised to the WHO 2000 standard population (9) and is given as rates by 100000 people and 95\% CIs. Differences in incidence rates between 2001-2005 and 2006-2011 were analysed by Poisson regression, accounting for age and gender. Categorical variables are presented with numbers and percentages, and continuous variables with means and s.D.s. For comparison between groups, Fisher's exact test was used for non-ordered categorical variables and the $t$-test was used for continuous variables. A two-sided $P<0.05$ was considered statistically significant.

The DevCan Software version 6.6.1 provided by the National Cancer Institute (Bethesda, MD, USA) $(10,11)$ was used for the calculations of the lifetime probability of PAs. The estimates were based on the incidence rate and mortality in 5-year age groups during 2001-2011 in VG County, Sweden. For all other analyses, the statistical software package Stata version 12.1 was used (StatCorp. 2012, Stata Statistical Software, release 12. College Station, TX, USA: StataCorp LP).

\section{Results}

\section{Demographics}

In the SPR, 983 patients from VG County were identified. Of these patients, 592 fulfilled the inclusion criteria. The remaining 391 patients were excluded because the diagnosis was established before 2001, they were not living in VG County at the time of diagnosis, or they were under the age of 18 at the time of diagnosis.

The demography of the patients included in the study is presented in Table 1. Fewer men were diagnosed with PAs than women, and men were older than women at diagnosis of PAs. The frequency of NFPA was higher in men than in women, whereas the frequency of prolactinomas was higher in women. More men than women had been surgically treated.

In total, 431 (73\%) patients were treated with surgery, irradiation or medication and were included in the treatment analysis, while the remaining 161 patients, all with NFPA, were followed clinically, biochemically and with MRI.

\section{Overall incidence of PAs}

The total incidence rate of PAs during the study period was 3.9/100 000 (CI 3.6-4.3). The overall SIR during 20012005 and 2006-2011 was similar, with an incidence rate ratio (IRR) of 1.09 (CI 0.92-1.28). 
Table 1 Demography of 592 patients diagnosed with pituitary adenomas (PAs) in the County of Västra Götaland between 2001 and 2011. The treatment of the 431 patients who underwent surgery, irradiation or were receiving medical treatment for PAs is also reported.

\begin{tabular}{l}
\hline \\
\hline Number (\%) \\
Mean age (s.D.) \\
Sub-types of PAs, $n(\%)$ \\
NFPA \\
Prolactinomas \\
Acromegaly \\
Cushing's disease \\
TSHomas \\
Gonadotrophinomas \\
Unknown \\
Macroadenomas, $n$ (\%) \\
Treatment \\
Surgery; no. of the cohort (\%) \\
Irradiation; no. of the cohort (\%) \\
Medical; no. of cohort (\%) \\
No treatment; no. of cohort (\%) \\
\hline
\end{tabular}

\begin{tabular}{c}
\hline All \\
\hline 592 \\
$51.7(18.3)$ \\
\\
$320(54.1)$ \\
$187(31.6)$ \\
$53(9.0)$ \\
$25(4.2)$ \\
$4(0.7)$ \\
$1(0.2)$ \\
$2(0.3)$ \\
$385(65)$ \\
$235 / 592(39.7)$ \\
$2 / 592(<1)$ \\
$194 / 592(32.8)$ \\
$161 / 592(27.2)$ \\
\hline
\end{tabular}

NFPA, non-functioning PA.

\section{Age- and gender-related incidence of PAs}

In men, the frequency of PAs increased with age, but decreased with age in women (Fig. 2A). Men had the highest incidence in the oldest age group, mainly due to NFPA (Fig. 2B). In women, the highest incidence of PAs was in the age range of 25-34 years, mainly due to a high incidence of prolactinomas (Fig. 2C). When patients with prolactinomas were excluded, the incidence of PAs in men increased with age, while the incidence in women remained unchanged (Fig. 2D).

The SIR of PAs in men was 3.3/100 000 (CI 2.9-3.7), which was lower than that in women (SIR 4.7/100 000 (CI 4.1-5.3)). IRR between men and women was 1.06 (CI 0.90-1.24).

The incidence rate in men increased during the study period. The SIR in men was 2.6/100 000 (CI 2.1-3.2) during 2001-2005 and 3.8/100 000 (CI 3.2-4.4) during 2006-2011, with a calculated IRR of 1.38 (CI 1.09-1.76). The corresponding figures in women were 5.2/100 000 (CI 4.2-6.1) during 2001-2005 and 4.3/100 000 (CI 3.6-5.1) during 2006-2011, with an IRR of 0.88 (CI 0.70-1.10). The IRR between women and men was 1.39 (CI 1.08-1.78) in 2001-2005 and 0.87 (CI 0.70-1.07) in 2006-2011 (Fig. 3A).

\section{Time trends of incidence}

The mean number (range) of patients diagnosed with PAs per year was 54 (42-69); 26 (17-36) in men and 28 (17-34) in women. The age-SIRs per 100000 people per year are

\begin{tabular}{c}
\hline Men \\
\hline $283(47.8)$ \\
$57.8(15.9)$ \\
\\
$185(65.4)$ \\
$62(21.9)$ \\
$27(9.5)$ \\
$6(2.1)$ \\
$1(0.4)$ \\
$1(0.4)$ \\
$1(0.4)$ \\
$232(82)$ \\
$144 / 283(50.9)$ \\
$1 / 283(<1)$ \\
$63 / 283(22.3)$ \\
$75 / 283(26.5)$ \\
\hline
\end{tabular}

\begin{tabular}{c}
\hline Women \\
\hline $309(52.2)$ \\
$46.2(18.7)$ \\
\\
$135(43.7)$ \\
$125(40.5)$ \\
$26(8.4)$ \\
$19(6.1)$ \\
$3(1.0)$ \\
0 \\
$1(0.3)$ \\
$153(50)$ \\
$91 / 309(29.5)$ \\
$1 / 309(<1)$ \\
$131 / 309(42.4)$ \\
$86 / 309(27.8)$ \\
\hline
\end{tabular}

\begin{tabular}{c}
\hline $\boldsymbol{P}$ \\
\hline$<0.001$ \\
$<0.001$ \\
\\
\\
\\
\\
\\
\\
\\
\\
\\
\end{tabular}

presented in Fig. 3. During the 11 years, the overall incidence rate remained unchanged. There was a $4.3 \%$ $(0.5 \%, 8.3 \% ; P=0.03)$ annual increase in incidence for men, but this was not the case for women, $-1.8 \% /$ year $(-5.2 \%, 1.7 \%$; $P=0.32$ ) (Fig. 3).

\section{Macro- and micro-adenomas}

Macroadenomas were found in 65\% $(n=385)$ of cases and microadenomas in 33\% $(n=198)$ (Table 1$)$. The information on size was not available in nine patients $(1.5 \%)$. Macroadenomas occurred in $82 \%$ of the men and $50 \%$ of the women (Table 1). SIR for macroadenomas was 2.3/100 000 (CI 2.0-2.5); 2.6/100 000 (CI 2.2-2.9) in men and 2.0/100 000 (CI 1.6-2.3) in women. SIR for microadenomas was 1.6/100 000 (CI 1.4-1.9); 0.7/100 000 (CI 0.5-0.8) in men and 2.6/100 000 (CI 2.1-3.1) in women.

\section{Incidences of subtypes of PAs}

NFPA - The most common subtype of PAs was NFPA, which accounted for $54 \%(n=320)$ of all cases and was more frequent in men than in women (Table 1). The incidence of NFPA increased with age (Fig. 2B). Of NFPA, $82 \%$ were macroadenomas, found in $88 \%$ of men and $73 \%$ of women. The SIR of NFPA was 1.8/100 000 (CI 1.6-2.0) and did not differ between men and women.

Prolactinomas - Prolactinomas were diagnosed in 32\% $(n=187)$ of cases (Table 1$) ; 37 \%$ of the prolactinomas were 

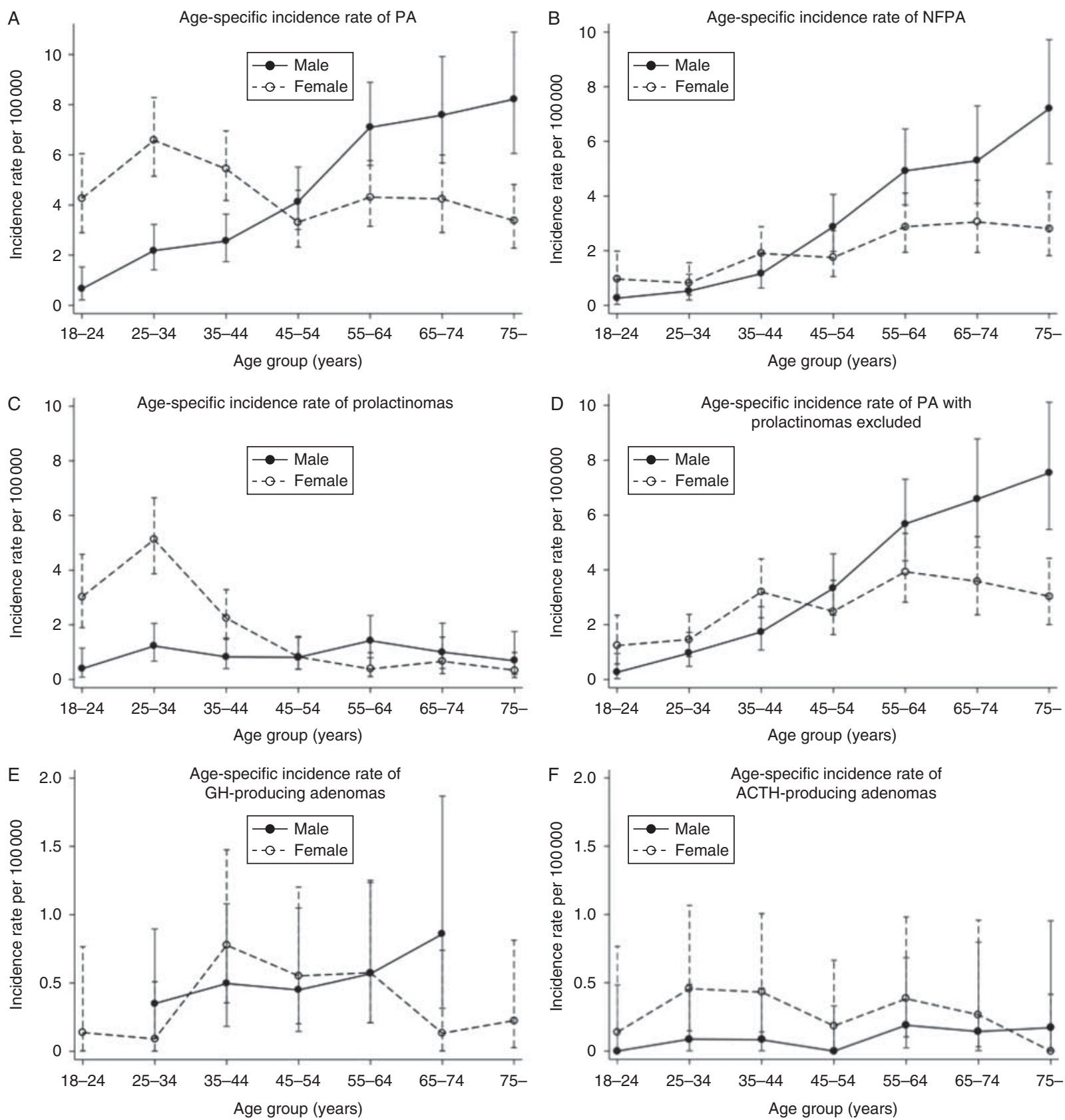

Figure 2

Age-specific incidence per 100000 people-years in patients with pituitary adenomas (PAs) diagnosed in Västra Götaland County between 2001 and 2011. (A) All PAs. (B) Non-functioning PA

(NFPA). (C) Prolactinomas. (D) PA with prolactinomas excluded, (E) GH-producing adenomas and (F) ACTH-producing adenomas.

macroadenomas, occurring in $68 \%$ of men and $22 \%$ in women. The SIR for prolactinomas was $1.6 / 100000$ (CI 1.3-1.9) and was higher in women (SIR 2.4/100 000 (CI 1.9-2.9)) than in men (SIR 0.9/100000 (CI 0.6-1.1)). Prolactinomas occurred most frequently

in premenopausal women, although the frequencies in men and postmenopausal women were similar (Fig. 2C).

Acromegaly - The third most common PA was GHproducing adenomas, accounting for 9\% $(n=53)$ of the 


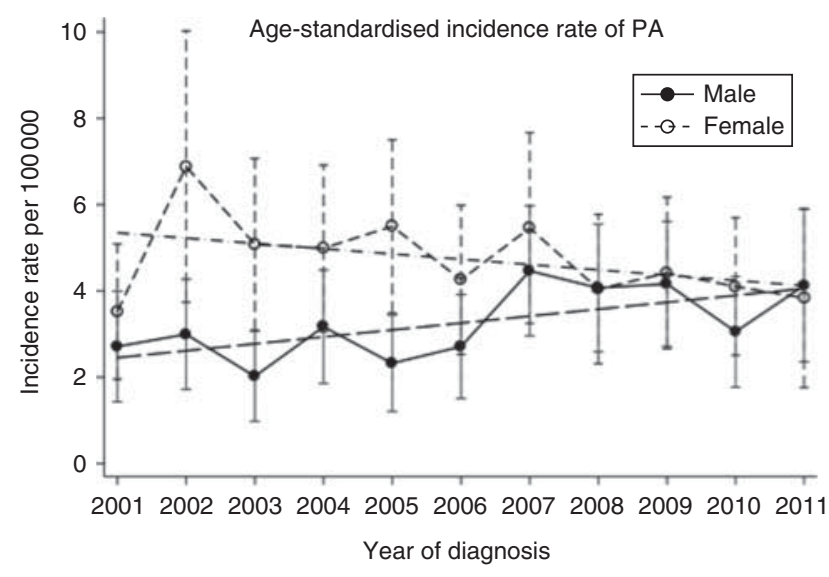

Figure 3

The incidence rate of pituitary adenomas (PAs) per 100000 inhabitants standardised to the world population per year in patients diagnosed between 2001 and 2011 in Västra Götaland, Sweden, in a targeted population of 1.6 million, including time trends in men and women.

cases (Table 1). SIR for acromegaly was $0.35 / 100000$ (CI $0.25-0.45)$ and was the same in men $(0.35 / 100000$ (CI 0.21-0.48)) and women (0.35/100 000 (CI 0.21-0.49)). GH-producing tumours were macroadenomas in $78 \%$ of men and $77 \%$ of women. The incidence rate of acromegaly was similar in all age groups (Fig. 2E).

Cushing's disease ACTH-producing PA accounted for $4 \%(n=25)$ of cases (Table 1); $28 \%$ of patients with Cushing's disease had a macroadenoma, with $33 \%$ in men and $26 \%$ in women. The SIR was $0.18 / 100000$ (CI 0.11-0.25) and was more common in women $(0.29 / 100000$ (CI 0.16-0.43)) than in men (0.07/100 000 inhabitants (CI 0.01-0.13)). The occurrence of Cushing's disease was evenly spread in the age groups (Fig. 2F).

TSHomas $~$ TSH-producing PA represented $0.7 \%(n=4)$ of all PAs (Table 1). The SIR for TSHomas was 0.03/100 000 (CI 0.00-0.05). All TSHomas were macroadenomas.

Gonadotrophinomas • Only one male patient was diagnosed with a clinically overt gonadotrophinoma during the study period.

Treatment $>$ Out of 592 patients, $73 \%(n=431)$ received treatment; the primary treatment was surgery in 55\% $(n=235)$, irradiation in $<1.0 \%(n=2)$ and medical treatment in $45 \%(n=194)$ (Table 1$)$.
Lifetime risk $~$ The lifetime risk of PAs was $0.27 \%$ (CI 0.24-0.31) in men and 0.29\% (CI 0.26-0.33) in women.

\section{Discussion}

As the largest incidence study of PAs, conducted during a period of frequent MRI use, this study revealed an overall incidence rate of 3.9/100 000, which was higher than what has been reported previously, before the MRI era (3). However, the incidence rate in this study corresponds well with a recent incidence study from Finland (8). The most common PAs were NFPA and prolactinomas, representing $86 \%$ of all cases. The results also agreed with the Finnish report (8), where prolactinomas and NFPA comprised $88 \%$ of the total number of PAs. As the SPR does not register information on the reason for diagnostic imaging, this study could not determine the proportion of incidentalomas. It is reasonable to assume that, as more sophisticated and sensitive diagnostic methods have been introduced during the last two decades, an increased detection of incidentally found PAs would be expected. Recent evidence for this has been presented by the Finnish report in which the incidence of symptomatic PAs did not increase; only more incidentalomas were found as a result of more frequent MRI use (8).

This study differed from the Finnish (8) and the Maltese (12) studies, in which the majority of PAs were prolactinomas rather than NFPA. The higher incidence of NFPA may reflect an increasing detection rate due to an increasing use of MRI during the 1990s. The lower frequency of prolactinomas may also be explained by the strict criteria used in this study, i.e. prolactin greater than three times above the upper reference range was required, which could result in some tumours being diagnosed, as NFPA would presumably be categorised as a prolactinoma in the other studies. Moreover, in those studies, the proportion of macroprolactinomas was significantly lower (27 and 18.5\%) than that in this study (37\%).

It is likely that some microprolactinomas are missing in the SPR, as prolactinomas may be diagnosed by gynaecologists who do not include patients in the SPR, and, thereby, the incidence of prolactinomas is underestimated. If it is assumed that the overall incidence of PAs is similar in the studies discussed previously $(8,12)$ and in the present one, an underestimation of prolactinomas in this study may be calculated by the difference in the mean incidence of prolactinomas in the Finnish and Maltese studies and by subtracting the incidence of prolactinomas in this study. This approximation results in an underestimation of 90 cases in this study compared with the 
other two studies. In this study, prolactinomas were defined according to a higher prolactin level than the two other studies. The consequence is that more patients may be defined as NFPA. If a similar calculation is done concerning this possible overestimation of NFPA when compared with the Finnish and Maltese studies, this overestimation represent 70 cases. Hence, as the sum of incidences of prolactinomas and NFPA in the Swedish, Finnish and Maltese studies is similar, this infers that the major reason for the lower incidence of prolactinomas is caused by the definition and that the number of lost cases due to incomplete coverage is relatively small.

There was a higher incidence of prolactinomas in the female population with an incidence peak during the fertile period. The incidence peak is probably a result of evident clinical manifestations, such as amenorrhoea, infertility and galactorrhoea, and lactotrophic cells in the pituitary gland might be sensitive to oestrogen (13). Therefore, the incidence peak might represent a true higher incidence instead of an effect of obvious symptomatology. After menopause, the incidence rate in women $(2.4 / 100000)$ was similar to that in men $(0.9 / 100000)$. Owing to milder clinical manifestations, the true incidence of prolactinomas in these groups might be underestimated (14) and may be the reason for the greater frequency of macroprolactinomas in men.

In this study, patients under the age of 18 were not included. In both the Finnish and Maltese studies, females between 10 and 19 years had a lower SIR (2.2 respectively 4.8) of prolactinomas compared with the overall SIR. It is likely that these proportions are similar in the Swedish population, which might have led to a slight overestimation of the incidence rate in the current study.

Macroadenomas (65\%) were more common than microadenomas (33\%). Macroadenomas have more often a tendency to grow, and therefore present clinically more often than microadenomas do (15). Some non-functioning microadenomas have no clinical manifestations, which might explain the higher microadenoma frequency in post-mortem studies (5) and contribute to the dominance of macroadenomas in this study. Therefore, the true incidence rate of microadenomas is probably higher than that observed in this study.

The frequent use of MRI as a diagnostic tool in the elderly population might account for the increase in NFPA with age, although the NFPA is probably equally distributed across the adult age range (16).

The incidence of ACTH-producing PA (0.18/100 000) was comparable to previous studies (0.17-0.24/100 000) $(8,17,18)$. Similarly, age at diagnosis, gender distribution
$(8,17,18)$ and the distribution in micro- and macroadenomas (19) were in accordance with previous studies. However, the number of patients with ACTH-producing PA $(n=25)$ was low and the results should be interpreted with caution.

The incidence rate for other hormone-producing PA (i.e. GH- and TSH-producing PA) was also in accordance with the previous study (8), but as the number of TSHomas was small, a valid SIR was difficult to maintain. The TSHoma incidence could be better evaluated from the national TSHoma incidence study in Sweden (20), which has an incidence of $0.15 /$ million inhabitants per year from 28 TSHoma found in 1990-2010.

In the current study, only primary treatment is reported, which explains the low frequency of irradiation. Irradiation was the standard treatment for patients with PAs until the late 1980s, when it was found that irradiation can be harmful to patients and result in an increased risk of vascular and cognitive impairment and the development of secondary tumours (21). This together with improved neurosurgical techniques may explain the low frequency of irradiation treatment in this cohort of patients. Thus, irradiation data on patients who received radiotherapy later on are not reported.

Although information on lifetime risk in patients with PAs is scarce, in this study, women had a higher lifetime risk for PAs than men, which was in agreement with reports from smaller cohorts (the Finnish and the Maltese cohort) with a higher incidence of PAs in females $(8,12)$.

The results of this large incidence study on PAs were strengthened by the health care system in Sweden that can identify each patient and exclude the risk of double registration. VG County has a large and well-defined population and patients' places of residence were verified through a search of the population register. However, this study did not cover all PAs, as only clinically evident PAs were included: sometimes clinically silent PAs (22) are only detected accidentally. In addition, the study did not cover all adult patients with prolactinomas due to lack of data from private practitioners. Recent case finding studies $(23,24)$ that provided a high coverage of patients have demonstrated a higher prevalence than previous studies and are complementary to this registry-based incidence study. Therefore, the true incidence rate may be higher than indicated.

As this is the largest study on PA incidence performed with modern diagnostic tools, the results are reliable, with the probable exception of microprolactinomas, and correspond well with recent studies with less than half the population size $(8,12)$. They can provide guidelines on 
the provision of medical resources, although further studies are warranted from different geographical areas to determine the true incidence of PAs.

\section{Declaration of interest}

The authors declare that there is no conflict of interest that could be perceived as prejudicing the impartiality of the research reported.

Funding

Grants supporting this study: the Sahlgren's Academy at University of Gothenburg and the LUA/Alf agreement at Sahlgrenska University Hospital, Göteborg, Sweden.

\section{Acknowledgements}

The authors are grateful to all clinics we visited for their courtesy and collaboration during data collection and to all clinics that collected patient data in the SPR. They are also grateful to Lise-Lott Norrman, MD, who has contributed with the initial ideas and guidance throughout the study.

\section{References}

1 Nilsson B, Gustavasson-Kadaka E, Bengtsson BA \& Jonsson B. Pituitary adenomas in Sweden between 1958 and 1991: incidence, survival, and mortality. Journal of Clinical Endocrinology and Metabolism 200085 1420-1425. (doi:10.1210/jcem.85.4.6498)

2 Mindermann T \& Wilson CB. Age-related and gender-related occurrence of pituitary adenomas. Clinical Endocrinology 199441 359-364. (doi:10.1111/j.1365-2265.1994.tb02557.x)

3 Burrow GN, Wortzman G, Rewcastle NB, Holgate RC \& Kovacs K. Microadenomas of the pituitary and abnormal sellar tomograms in an unselected autopsy series. New England Journal of Medicine 1981304 156-158. (doi:10.1056/NEJM198101153040306)

4 Teramoto A, Hirakawa K, Sanno N \& Osamura Y. Incidental pituitary lesions in 1,000 unselected autopsy specimens. Radiology 1994193 161-164. (doi:10.1148/radiology.193.1.8090885)

5 Faglia G. Epidemiology and pathogenesis of pituitary adenomas. Acto Endocrinologica 1993129 (Suppl 1) 1-5.

6 Regal M, Paramo C, Sierra SM \& Garcia-Mayor RV. Prevalence and incidence of hypopituitarism in an adult Caucasian population in northwestern Spain. Clinical Endocrinology 200155 735-740. (doi:10.1046/j.1365-2265.2001.01406.x)

7 Ezzat S, Asa SL, Couldwell WT, Barr CE, Dodge WE, Vance ML \& McCutcheon IE. The prevalence of pituitary adenomas: a systematic review. Cancer 2004101 613-619. (doi:10.1002/cncr.20412)

8 Raappana A, Koivukangas J, Ebeling T \& Pirila T. Incidence of pituitary adenomas in northern Finland in 1992-2007. Journal of Clinical Endocrinology and Metabolism 201095 4268-4275. (doi:10.1210/ jc.2010-0537)

9 Ahmad OE, Lopez AD, Murray CJL, Lozano R \& Inoue M Age standardization of rates: a new WHO standard. GPE discussion paper series 31, Geneva: World Health Organization, 2000
10 Fay MPPR, Cronin KA, Le C \& Feuer EJ. Age-conditional probabilities of developing cancer. Statistics in Medicine 200322 1837-1848. (doi:10.1002/sim.1428)

11 Fay M. Estimating age conditional probability of developing disease from surveillance data. Population Health Metrics 2004276. (doi:10.1186/1478-7954-2-6)

12 Gruppetta M, Mercieca C \& Vassallo J. Prevalence and incidence of pituitary adenomas: a population based study in Malta. Pituitary 2013 16 545-553. (doi:10.1007/s11102-012-0454-0)

13 Maurer RA. Estradiol regulates the transcription of the prolactin gene. Journal of Biological Chemistry 1982257 2133-2136.

14 Casanueva FF, Molitch ME, Schlechte JA, Abs R, Bonert V, Bronstein MD, Brue T, Cappabianca P, Colao A, Fahlbusch R et al. Guidelines of the Pituitary Society for the diagnosis and management of prolactinomas. Clinical Endocrinology 200665 265-273. (doi:10.1111/j.1365-2265.2006.02562.x)

15 Fernandez-Balsells MM, Murad MH, Barwise A, Gallegos-Orozco JF, Paul A, Lane MA, Lampropulos JF, Natividad I, Perestelo-Pérez L, Ponce de León-Lovatón PG et al. Natural history of nonfunctioning pituitary adenomas and incidentalomas: a systematic review and metaanalysis. Journal of Clinical Endocrinology and Metabolism 201196 905-912. (doi:10.1210/jc.2010-1054)

16 Freda PU, Beckers AM, Katznelson L, Molitch ME, Montori VM, Post KD, Vance ML \& the Endocrine Society. Pituitary incidentaloma: an endocrine society clinical practice guideline. Journal of Clinical Endocrinology and Metabolism 201196 894-904. (doi:10.1210/ jc.2010-1048)

17 Lindholm J, Juul S, Jorgensen JO, Astrup J, Bjerre P, Feldt-Rasmussen U, Hagen C, Jørgensen J, Kosteljanetz M, Kristensen L et al. Incidence and late prognosis of Cushing's syndrome: a population-based study. Journal of Clinical Endocrinology and Metabolism 200186 117-123.

18 Bolland MJ, Holdaway IM, Berkeley JE, Lim S, Dransfield WJ Conaglen JV, Croxson MS, Gamble GD, Hunt PJ \& Toomath RJ. Mortality and morbidity in Cushing's syndrome in New Zealand. Clinical Endocrinology 201175 436-442. (doi:10.1111/j.1365-2265. 2011.04124.x)

19 Valassi E, Santos A, Yaneva M, Toth M, Strasburger CJ, Chanson P, Wass JA, Chabre O, Pfeifer M, Feelders RA et al. The European Registry on Cushing's syndrome: 2-year experience. Baseline demographic and clinical characteristics. European Journal of Endocrinology 2011165 383-392. (doi:10.1530/EJE-11-0272)

20 Onnestam L, Berinder K, Burman P, Dahlqvist P, Engstrom BE, Wahlberg J \& Filipsson Nyström H. National incidence and prevalence of TSH-secreting pituitary adenomas in Sweden. Journal of Clinical Endocrinology and Metabolism 201398 626-635. (doi:10.1210/jc.2012-3362)

21 Ecemis GC, Atmaca A \& Meydan D. Radiation-associated secondary brain tumors after conventional radiotherapy and radiosurgery. Expert Review of Neurotherapeutics 201313 557-565. (doi:10.1586/ern.13.37)

22 Cooper $\mathrm{O} \&$ Melmed S. Subclinical hyperfunctioning pituitary adenomas: the silent tumors. Best Practice \& Research. Clinical Endocrinology \& Metabolism 201226 447-460. (doi:10.1016/j.beem. 2012.01.002)

23 Fernandez A, Karavitaki N \& Wass JA. Prevalence of pituitary adenomas: a community-based, cross-sectional study in Banbury (Oxfordshire, UK). Clinical Endocrinology 201072 377-382. (doi:10.1111/j.1365-2265. 2009.03667.x)

24 Daly AF, Rixhon M, Adam C, Dempegioti A, Tichomirowa MA \& Beckers A. High prevalence of pituitary adenomas: a cross-sectional study in the province of Liege, Belgium. Journal of Clinical Endocrinology and Metabolism 200691 4769-4775. (doi:10.1210/jc.2006-1668)
Received 18 February 2014

Revised version received 9 July 2014

Accepted 1 August 2014 